\title{
Instant Blood-mediated Inflammatory Reaction During Islet Transplantation: The Role of Toll-like Receptors Signaling Pathways
}

\author{
K. Vivot, A. Langlois, N. Jeandidier, W. Bietiger, M. Pinget, JP. Gies, and S. Sigrist
}

\begin{abstract}
The instant blood-mediated inflammatory reaction (IBMIR) leads to massive destruction of transplanted islets. Islet isolation and time of culture may elicit the release of potent activators of Toll-like receptors (TLRs) signaling pathways during IBMIR. This work sought to evaluate the role of TLR signaling pathways to mediate inflammatory reactions. Isolated rat pancreatic islets were cultured for 12,24 , or 48 hours. Their viability was assessed by fluorescein diacetate/propidium iodide and their functionality, by glucose stimulation tests. Endotoxin levels were quantified using the Limulus Amebocyte Lysate assays. After RNA extraction and reverse transcription, we performed polymerase chain reaction (PCR) arrays. Samples obtained immediately after isolation were defined as controls. Eighty-four genes belonging to the TLR signaling pathways, were compared with control samples. After culture, islets were viable and functional with low endotoxin levels $(<0.1$ endotoxin units $/ \mathrm{mL})$ showed TLR activation not due to exogenous contamination. Analysis of PCR arrays highlighted significant up-regulation of TLR-2. After 24 hours of culture, TLR-2 was up-regulated to $6.8 \pm 0.6$-fold $(P<.001)$ compared with controls but decreased to $4.3 \pm 1.4$-fold after 48 hours. In the same way, expression of myeloid differentiation primary response gene 88 (Myd88) was significantly up-regulated (3.2 \pm 0.4 -fold $[P<.001])$ compared with controls. After 12 hours of culture, interleukin-10 gene expression was significantly up-regulated at $11.6 \pm 3.7$ - fold $(P<.05)$, reaching $17.5 \pm 8.3$ after 24 hours. Finally, the cyclo-oxygenase- 2 gene expression was up-regulated to $509 \pm$ 67.1-fold $(P<.05)$ after 12 hours of culture. These data confirmed the implication of TLR signaling pathways in early inflammatory events.
\end{abstract}

$\mathbf{R}^{\mathrm{s}}$ ESTORATION of endogenous insulin by islet transplantation is an attractive treatment for Type 1 diabetes mellitus. ${ }^{1}$ However, the instant blood-mediated inflammatory reaction (IBMIR) destroys approximately $60 \%$ of pancreatic islets immediately after transplantation. ${ }^{2}$ Many reports suggest the implication of the Toll-like receptor (TLR) signaling pathway in IBMIR. ${ }^{3-5}$

TLRs, which belong to the pattern pathogen receptors family, were originally known to bind pathogen-associated molecular patterns. Recently, many reports suggest that TLRs bind endogenous ligands called Alarmins that are defined as inflammatory mediators that are generated and released after tissue damage. ${ }^{6}$ Thus, early events of islet isolation and culture can elicit the release of potent activators of IBMIR. The purpose of this work was to evaluate in vitro the role of TLR signaling pathways to mediate inflammatory reactions before transplantation.

\section{MATERIALS AND METHODS}

Rat pancreatic islets were isolated from male Wistar rats (Centre d'élevage Depré, Saint Doulchard, France) weighting 250 to $300 \mathrm{~g}$ by distending the pancreatic duct with collagenase (Sigma, St Quentin Fallavier, France), as previously described. ${ }^{7}$ Islets were cultured for 12, 24, and 48 hours in M199 medium (Gibco, Fischer, Illkirch, France) containing 10\% heat-inactivated fetal calf serum (Lonza, Verviers, Belgique) and $1 \%$ antibiotic-antimycotic (Gibco). After isolation, 20 pancreatic islets cells were obtained by handpicking to evaluate viability and functionality. RNA was

From the Centre Européen d'Étude du Diabète (K.V., A.L., N.J., W.B., M.P., S.S), Strasbourg, France and the Université de Strasbourg, Strasbourg, France (N.J., M.P.); and Faculté de Pharmacie (J.P.G.), Illkirch, France.

Address reprint requests to Kevin Vivot, Centre Européen d'Étude du Diabète, Boulevard René Leriche, 67200 Strasbourg, France. E-mail: k.vivot@ceed-diabete.org 360 Park Avenue South, New York, NY 10010-1710 

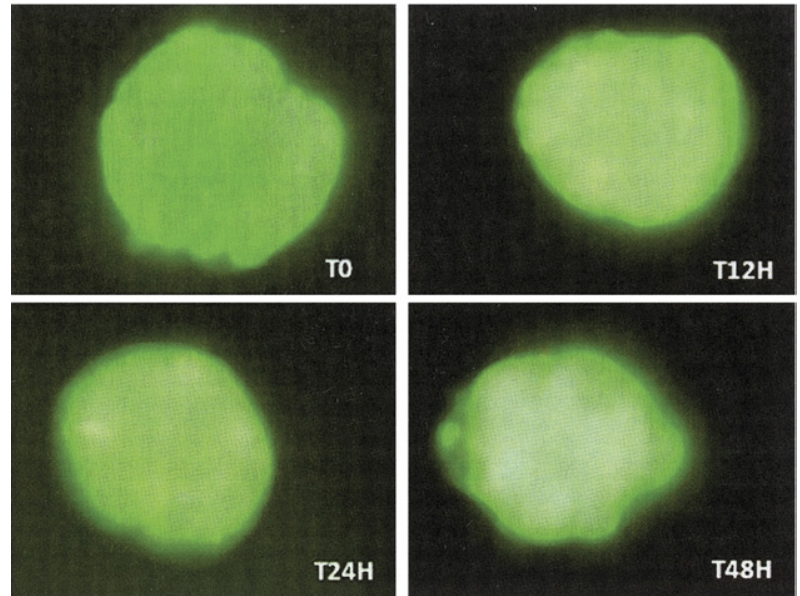

Fig 1. Viability of rat pancreatic islets using fluorescein diacetate/propidium iodide staining.

extracted from the remaining islet cells for reverse-transcription to complementary DNA. Samples extracted immediately after isolation were defined as the controls. To detect the eventual presence of endotoxin contaminants in the culture supernates, we used the Endpoint Chromogenic LAL, QCL-1000 ${ }^{\mathrm{TM}}$ assay (Lonza) following the manufacturer's instruction.

Islet viability was determined using fluorescein diacetate; (Sigma) and propidium iodide. To evaluate functionality, cells were consecutively stimulated for 90-minute periods in Krebs buffer containing $3.3 \mathrm{mmol} / \mathrm{L}$ glucose (period one) and $27.5 \mathrm{mmol} / \mathrm{L}$ glucose (period two). After cell lysis, the amount of protein was evaluated according to the Bradford method with insulin concentrations determined by enzyme-linked immunosorbent (Mercodia, Uppsala, Sweden). Results were expressed as the ratio of insulin/ total proteins. Polymerase chain reaction (PCR) arrays (SABiosciences, Frederick, Maryland, USA) were performed following the manufacturer's instructions.
Data were expressed as mean values \pm standard error of the mean Student $t$ tests were used to evaluate statistical significance with $P$ values $<.05$ considered to be significant.

\section{RESULTS}

The islets were viable (Fig 1) and functional (data not shown) without endotoxin contamination, namely, levels in all culture supernates were less than 0.1 units $/ \mathrm{mL}$.

Among the 84 screened genes, the expressions of six genes were modulated during the culture. TLR-2 was up-regulated compared to controls during the culture. At 12 hours, TLR-2 expression was significantly increased to $5.5 \pm 1.6$ fold $(P<.05)$ compared with controls being highest at 24 hours, namely $6.8 \pm 0.6$ increase $(P<.001)$. In the same way, at 24 hours the gene expression of myeloid differentiation primary response gene 88 (Myd88) was significantly up-regulated to $3.2 \pm 0.4$-fold $(P<.001)$ (Fig 2A).

Cyclooxygenase-2 (COX-2) gene expression decreased in a time-dependent manner. After 12 hours of culture, gene expression was up-regulated $509 \pm 67.1$ fold $(P<.05)$ decreasing to $89.9 \pm 56.3$-fold at 48 hours of culture (Fig 2B). Moreover, cytokine and chemokine gene expressions were also up-regulated in time-dependent manner including the COX-2 gene. The transcript level was highest at 12 hours of culture. For example, interleukin (IL)-10 and CXCL10 gene expressions were increased $11.6 \pm 3.7$-fold $(P<.05)$ and $11.8 \pm 2.6(P<.05)$-fold, respectively at 48 hours of culture, the change in IL-10 and CXCL10 reached only $6.8 \pm 5.5$ and $1.23 \pm 0.3$-fold, respectively (Figs $2 \mathrm{C}$, 2D). As expected, IL-6 gene expression was dramatically increased at 12 hours to $1757.5 \pm 824.6$-fold decreasing to $110.1 \pm 92.6$-fold at 48 hours of culture.
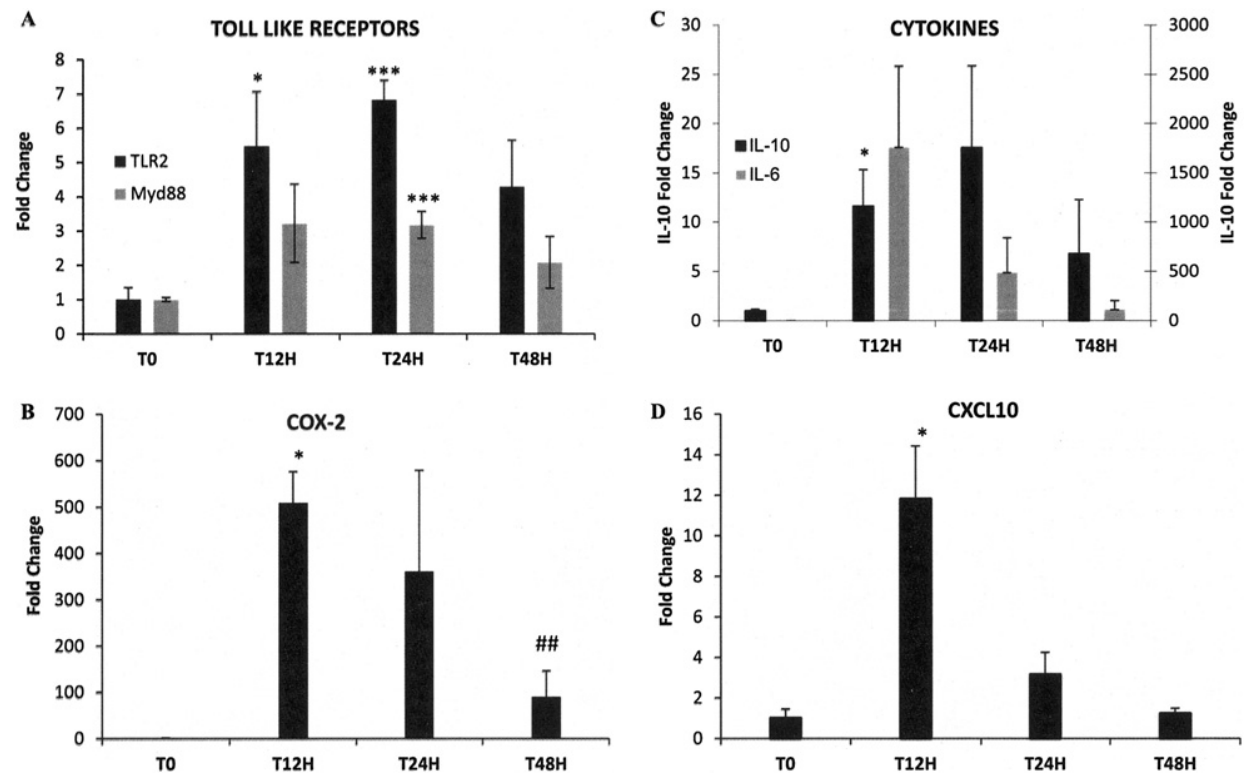

Fig 2. Fold regulation of (A) Toll-like receptors, (B) cyclooxygenase-2 (COX-2), (C) cytokines, (D) CXCL10, and gene expression of Rat pancreatic islet after $0,12,24$, and 48 hours of culture. 


\section{DISCUSSION}

Finally, these data confirmed the involvement of TLR signaling pathways in the early events before transplantation. However, an understanding of molecular mechanisms and potent activators of IBMIR could yield improved islet transplantation.

\section{REFERENCES}

1. Shapiro AM: Islet transplantation-the imperative need for continued clinical trials. Nat Clin Pract Nephrol 4:662, 2008

2. Barshes NR, Wyllie S, Goss JA: Inflammation-mediated dysfunction and apoptosis in pancreatic islet transplantation: implications for intrahepatic grafts. J Leucocyte Biol 77:587, 2005

3. Goldberg A, Parolini M, Chin BY, et al: Toll-like receptor 4 suppression leads to islet allograft survival. FASEB J 21:2840, 2007

4. Gao Q, Ma LL, Gao X: TLR4 mediates early graft failure after intraportal islet transplantation. Am J Transplant 10:1588, 2010

5. Krüger B, Yin N, Zhang N, et al: Islet-expressed TLR2 and TLR4 sense injury and mediate early graft failure after transplantation. Eur J Immunol 40:2914, 2010

6. Erridge C: Endogenous ligands of TLR2 and TLR4: agonist or assistants? 87:989, 2010

7. Sutton R, Peters M, McShane P, et al: Isolation of rat pancreatic islets by ductal injection of collagenase. Transplantation 42:689, 1986 